\title{
How to Ensure Nutrition Security - A Critical Review
}

\section{Ojha $A^{1}$, Singh $A^{1 *}$, Sharma $A^{2}$ and Upadhyay $A^{3}$}

${ }^{1}$ Assistant Professor, Department of Food Science \& Technology, National Institute of Food Technology Entrepreneurship \& Management (NIFTEM), Kundli, Sonepat, Haryana, India

2Scientist, ZTM \& BPD Unit, Indian Agricultural Research Institute (IARI), Pusa, New Delhi, India

${ }^{3}$ Professor \& Head, Department of Food Science \& Technology, National Institute of Food Technology Entrepreneurship \& Management (NIFTEM), Kundli, Sonepat, Haryana, India

*Corresponding author: Anurag Singh, Assistant Professor, Department of Food Science \& Technology, National Institute of Food Technology Entrepreneurship \& Management (NIFTEM), Kundli, Sonepat, Haryana, India, Tel: +918199990641; Email: anurag.niftem@gmail.com

\section{Abstract}

Malnutrition in its different forms like under-nutrition, deficiencies of micronutrient and overweight or obesity, impress high economic and social costs on developing countries at all income levels. Actually improving nutrition and reducing these costs begin with agriculture practices and food systems - supply chain. The social cost of malnutrition that is measured by the "disability-adjusted life years" lost to child and maternal malnutrition and to overweight and obesity, is very high. Beyond the social cost, the cost to the global economy caused by malnutrition, as a result of lost productivity and direct health care costs, could account for as much as 5 percent of global gross domestic product (GDP). The size of problem is like malnutrition affects all countries and one in three people on the earth. The good news is - we know how to curb this menace of malnutrition and can achieve the goals of nutrition security through Supplementation, Fortification, Bio-fortification in agriculture and Dietary diversification through Awareness.

Keywords: Nutrition Security; Malnutrition; SAM; MAM; Supplementation; Fortification

Abbreviations: GDP: Gross Domestic Product; GIT: Gastro-Intestinal Tract; RUTF: Ready-to-Use Therapeutic Food; RUSF: Ready to Use Supplementary Foods; LNS: Lipid Based Nutrient Supplement; FSSAI: Food Safety and
Standards Authority of India; KDCMPUL: Kaira District Co-operative Milk Producers Union Limited; UNICEF: United Nations Children's Fund. 


\section{Food Science and Nutrition Technology}

\section{Introduction}

Nutrition security is an integral part of food security with two more dimensions. According to FAO " Food security exists when all people, at all times, have physical, social and economic access to sufficient, safe and nutritious food which meets their dietary needs and food preferences for an active and healthy life" [1], while the committee on world food security has defined nutritional security as " All people, at all times, have physical, social and economic access to food, which is safe and consumed in sufficient quantity and quality to meet their dietary needs and food preferences and is supported by an environment of adequate sanitation, health services, care, for an active and healthy life" [2]. In the description of Nutrition security, emphasis is given on the word Quality - means Nutrition availability of all micronutrients - along with a good support of health care services. For example in a geographic area the people are consuming sufficient quantity and quality (nutrition) of food but are suffering from inflammation of gut (GIT- Gastro-intestinal Tract) which hinders bio- absorption of nutrients than the purpose may not be addressed until the geographic area will have adequate sanitation and health services.

Malnutrition is an abnormal physiological condition caused by inadequate, unbalanced or either low or excessive consumption of the macronutrients that provide dietary energy (Proteins, Fat or Carbohydrates) and the micronutrients (vitamins and minerals) those are essential for physical as well as cognitive growth and development. Good nutrition both, depends on and contributes to good health. Micronutrient malnutrition is defined as being deficient in one or more vitamins and minerals of importance for human health. It is an outcome of inappropriate dietary composition and disease.

The FAO publication - The state of food and Agriculture, 2013 [3] states that Malnutrition in all its forms - undernutrition, micronutrient deficiencies, and overweight or obesity - imposes unacceptably high economic and social costs on countries at all income levels. Food systems for better nutrition argue that improving nutrition and reducing these costs must begin with food and agriculture.

Available cross-country evidence on child nutritional status consistently shows that, on average, children in urban areas are better nourished than children in rural areas. The most recent data compiled by UNICEF (2013) shows that in 82 out of 95 developing countries for which data are available, the prevalence of child underweight is higher in rural areas than in urban areas. Evidence from India indicates that the rural-urban divide may also hold for adults. A Prevalence of adult underweight was found around 23 percent in the slum areas of eight Indian cities, while the prevalence in rural areas in the same states was close to 40 percent. Combination of laborious farm employment and weaker access to education and health services jointly contribute to rural adult nutrition indicators being substantially worse than those of urban slum populations.

\section{Magnitude of the Problem}

FAO's most recent estimate point out that around 900 million people (12.5 percent of the world's population) are undernourished in terms of kcals intake, yet these figures represent only a fraction of the global load of malnutrition. An estimated 26 percent of the world's children are stunted, 2 billion population experience with one or more micronutrient deficiencies 1.4 billion people are overweight and of whom 500 million are overweight. Many countries are burdened by numerous types of malnutrition, which may coexist within the same geography, and individual.

The consequence of this hidden hunger (nutrition security) can be seen throughout life. A vicious cycle mentioned in Figure 1 clearly shows that if a pregnant woman with under nutrition, will give birth to a baby with low birth weight and impaired mental development than throughout his or her (baby) life He or she will suffer in all the stages - Child, adolescent, adult and elderly with multiple disorder and diseases. This vicious cycle will again give birth a baby of lower birth weight and so on. 


\section{Food Science and Nutrition Technology}

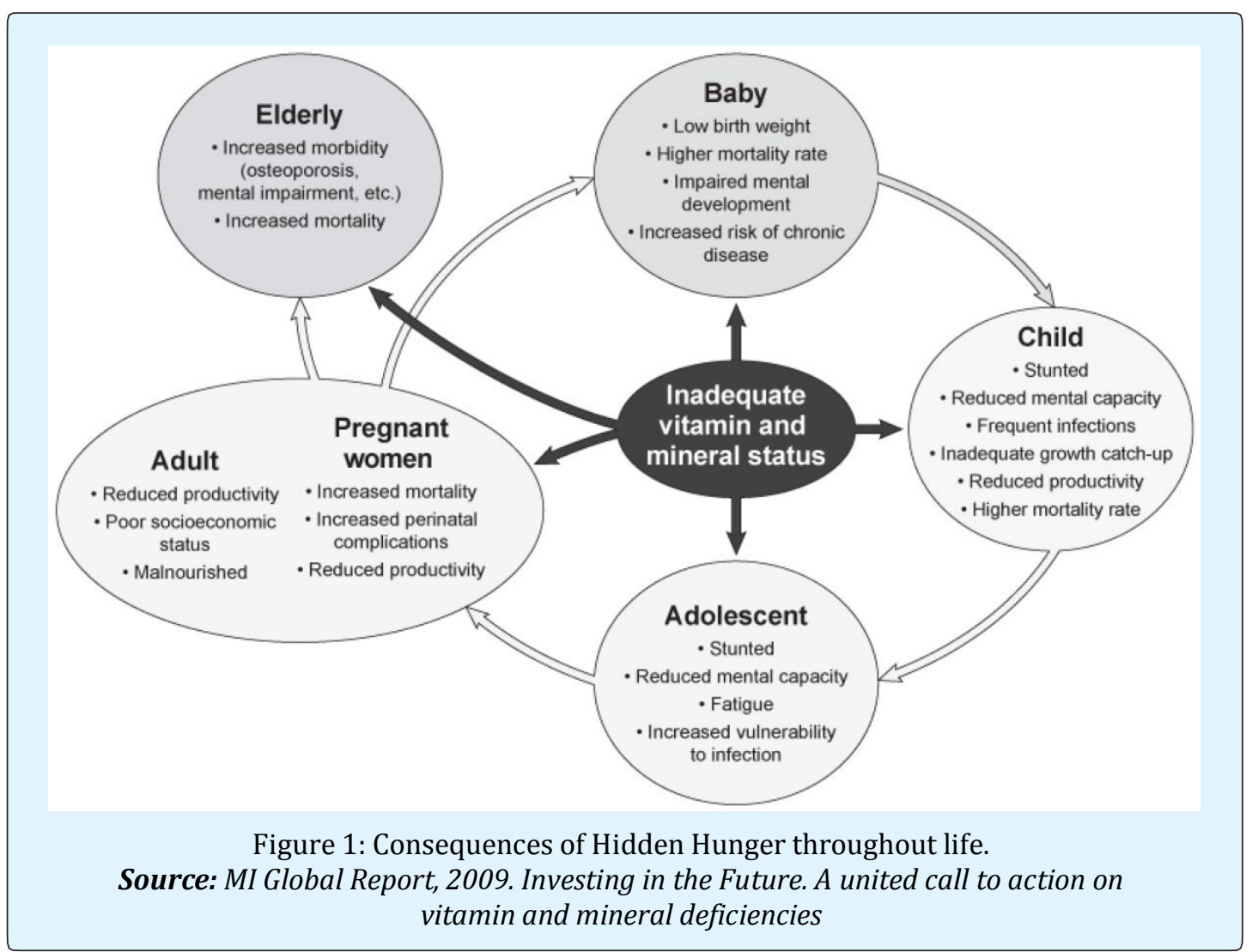

The proposed solutions to curb this vicious cycle are as follows:

\section{Supplementation}

Providing pills dense with micronutrients is a very easy way to fulfill the nutritional requirement. For example Iron tablets are distributed to avoid anemia especially in females.

\section{Fortification}

Fortifying the existed food products with various micronutrients to develop RUTF (ready-to-use therapeutic food), RUSF (Ready to use supplementary foods), LNS (Lipid based nutrient supplement) etc. is being practiced in a country like India to overcome nutritional deficiency.

\section{Bio-Fortification}

Biofortification is an easy and cost-effective method to deliver micronutrients to the populations having limited access to diverse diets and other micronutrient interventions available. Increasing the density of vitamins and minerals in a crop through plant breeding, transgenic techniques, or agronomic practices can fulfill the need of the human body for these micro-nutrients by consuming these crops. Bio-fortified staple crops, when consumed regularly, will generate measureable improvements in human health and nutrition [4].

\section{Dietary Diversification}

The awareness about - we eat food or we plant food or we have our platter for nutrition and not only energy needs to be created. The diversification in diet can be achieved by outreach activities to generate awareness in the masses about importance of complete diet and problems associated with malnutrition. Dietary diversity is a key determinant of nutritional outcomes, but the consumption of nutrient-dense foods is very sensitive to income and price shocks, especially for low-income consumers. Protecting the nutritional quality of diets not just the adequacy of staple food consumption - should be a priority for policy-makers

\section{Initiatives taken in India}

The fundamental role of agriculture is to produce food, what we eat and to generate income for the farmers and 


\section{Food Science and Nutrition Technology}

value chain. Other than this, agriculture and the food value chain - from agri inputs and production, through processing, storage, transport and distribution, till consumption - can contribute much more to the eradication of malnutrition. All forms of malnutrition share a common cause: inappropriate diets that provide inadequate, unbalanced or excessive macronutrients and micronutrients. The only sustainable means of addressing malnutrition is through the consumption of a high quality, diverse diet that provides adequate but not excessive energy. Food systems determine the availability, affordability, diversity and quality of the food supply and thus play a major role in shaping healthy diets. Different agencies and schemes those are working to develop healthy diets for Indian populations and healthy products developed are as follows:

\section{FSSAI}

The Food Safety and Standards Authority of India (FSSAI), a statuary regulatory body working under Ministry of Health and family welfare has come up with a solution for fortification of some article of foods under Food Safety and Standards (Fortification of Foods) Regulations, 2016 [5].

a. Milk - Fortifying with Vit- A and D

b. Rice - Iron, folic acid, vit- B12, zinc, vit A, thiamine, riboflavin, niacin, pyridoxine.

c. Salt - as double fortified salt - Iodine and Iron

d. Wheat Flour - Iron, Folic Acid, Vit B12, Zinc, Vit A, Thiamine, Riboflavin, Noacin and Pyridoxine.

e. Vegetable oil- Vit A and D.

The Authority has identified these articles of food with greater relevance as all the articles selected have greater penetration in terms of consumption by masses.

\section{Bal Amul}

The Kaira District Co-operative Milk Producers Union Limited (KDCMPUL) popularly known as Amul Dairy in Anand, having a tag line "The Taste of India", is setting up a plant to produce ready-to-use therapeutic food (RTUF) for millions of severely malnourished children in the country on a non-commercial basis. Both, the Gujarat government and Government of Bihar have approached Amul and procured the newly developed RTUF to be manufactured in the plant approved by United Nations Children's Fund (UNICEF). Bal Amul is specifically developed for children above six months. The therapeutic food made by Amul has milk solids and fortified with vitamins, minerals and protein. Already Amul is supplying Bal Amul to Sudan and Afghanistan. [6].

\section{Doodh Sanjeevani Yojana}

It was a project of Rs. 117 million carried out at Gujarat. Gujarat Co-operative Milk Marketing Federation which markets the brand Amul-GCMMF, has supplied a flavored milk called as Sanjeevani. This is a UHT processed flavored milk, which is shelf stable upto 120 days and is packed in pouches as market milk. The students get 200 $\mathrm{ml}$ of milk in schools everyday fortified with added $3 \%$ fat, $24 \mathrm{~g}$ carbohydrate, protein 7 g., vitamin A 500 I.U., vitamin D 40 I.U. This is not the only example of Govt. scheme for providing nutrition but the importance of this instance is like - with technological advances now the flavored milk can be treated by UTH process with a low packaging cost and higher shelf life at room temp.

\section{Sagar Poshan}

This is a kind of lipid based nutrient supplement developed by University of Illinois in association with Dudhsagar Dairy, Mansinhbhai Institute of Dairy \& Food Technology (MIDFT), India and National Institute of Food Technology Entrepreneurship \& Management (NIFTEM). Lipid-based nutrient supplements (LNS) are ready to use energy and nutrient dense food products that are suitable to complement most food rations, especially due to their longer shelf life and convenience. Sagar poshan was made with all the ingredients from Indian bowl and is at par with any of the RUTF. In this LNS, Omega 3: Omega 6 fatty acids are balanced. Balanced Omega-6/Omega-3 polyunsaturated fatty acids (PUFA) are beneficial for children suffering from SAM or MAM, as they serve as precursors for eicosapentaenoic acid (EPA) and docosahexaenoic acid (DHA), which plays a significant role in modulation of tissue inflammation, immune function, and overall neural development [7]. In addition, omega-3 PUFA modulates intestinal inflammation, common in malnourished children, that results in malabsorption of nutrients from the diet $[8,9]$. Currently, there are no specifications for omega-6/omega-3 PUFA ratio in LNS, however, the importance of balanced PUFAs in LNS is now well recognized [10].

\section{Iron Fortified Rice (IFR)}

Food Chemistry and Technology Laboratory at IIT Kharagpur has developed a technology to fortify iron and folic acid in rice. The technology utilizes broken rice powder that is fortified with iron as well as folic acid and then extruded to form rice like kernels. These iron fortified rice kernels can be mixed with normal rice in a ratio of 1:100. This mixture provides $\mathbf{5 0}$ per cent of recommended daily allowance (RDA) of Iron. 


\section{Food Science and Nutrition Technology}

This technology can also be used to fortify rice with other micro nutrients, as well. The technology is cost effective too. The cost of fortification has been estimated by IITKharagpur to be upto 80 paise per kg of rice [11].

\section{Conclusion}

Finally at the end it is worthwhile to mention that curbing the vicious cycle is not a rocket science. We can also improve our own traditional food articles like chaas (butter milk), traditional bakery items like Nankhatai, traditional sweets and energy bars with micronutrients. A country can formulate its own standards through statuary/regulatory body for fortification of articles of food, either in public distribution system (PDS) or effective distribution system. The malnutrition burden in a country changes rapidly with the transformation of the food system. Policy-makers must therefore understand the specific nature of the malnutrition problem to design interventions throughout the food system. Up-to-date data and analysis are necessary to support decisionmaking. A small step in fortification and dietary diversification will lead to giant leap towards Nutritional Security.

\section{References}

1. Food and Agriculture Organization of the United Nations (FAO) 1996. The state of food and agriculture.

2. Committee on World Food Security (CFS) 2012. Report of the 39th session, 15-20 Oct, 2012. FAO, Rome, Italy.
3. FAO (2013) Food systems for Better Nutrition. The state of food and agriculture.

4. Bouis HE, Saltzman A (2017) Improving nutrition through biofortification: A review of evidence from Harvest Plus, 2003 through 2016, Global Food Security 12: 49-58.

5. Food safety and standards (fortification of foods) Regulation, 2016.

6. 'Bal Amul' to help Bihar fight malnutrition. Times of India Life, Feb 10, 2016.

7. Calder PC (2006) n-3 Polyunsaturated fatty acids, inflammation, and inflammatory diseases. American Journal of Clinical Nutrition 83(6):1505-1519.

8. Sen SG, Mohammed MH, Ghosh TS, Kanungo S, Nair $\mathrm{GB}$, et al. (2011) Metagenome of the gut of a malnourished child. Gut Pathogens 3: 7.

9. Teitelbaum JE, Allan Walker W (2001) Review: the role of omega 3 fatty acids in intestinal inflammation. The Journal of Nutritional Biochemistry 12(1): 21-32.

10. Brenna JT, Akomo P, Bahwere P, Berkley J, Calder PC, et al. (2015). Balancing omega-6 and omega-3 fatty acids in ready-to-use therapeutic foods (RUTF). BMC Medicine 13: 1-4.

11. Indigenously developed iron-fortified rice premix to address anemia, Article published on line in business standard (2017). 